TRANSACTIONS OF THE

AMERICAN MATHEMATICAL SOCIETY

Volume 358, Number 8, Pages 3459-3472

S 0002-9947(05)03872-9

Article electronically published on September 22, 2005

\title{
A GEOMETRIC CHARACTERIZATION OF INTERPOLATION IN $\hat{\mathcal{E}}^{\prime}(\mathbb{R})$
}

\author{
XAVIER MASSANEDA, JOAQUIM ORTEGA-CERDÀ, AND MYRIAM OUNAÏES
}

\begin{abstract}
We give a geometric description of the interpolating varieties for the algebra of Fourier transforms of distributions (or Beurling ultradistributions) with compact support on the real line.
\end{abstract}

\section{INTRODUCTION}

Let $\mathcal{E}(\mathbb{R})$ be the space of smooth functions in $\mathbb{R}$ and let $\mathcal{E}^{\prime}(\mathbb{R})$ be its dual, the space of distributions with compact support on $\mathbb{R}$. It is well known that the space $\hat{\mathcal{E}}^{\prime}(\mathbb{R})$ of Fourier transforms of distributions in $\mathcal{E}^{\prime}(\mathbb{R})$ coincides with the algebra of entire functions $f$ such that

$$
|f(z)| \leq C(1+|z|)^{A} e^{B|\operatorname{Im} z|},
$$

where $A, B, C>0$ may depend on $f$ (see BrGa95, Theorem 1.4.15]).

A discrete sequence $\Lambda \subset \mathbb{C}$ is called $\hat{\mathcal{E}}^{\prime}(\mathbb{R})$-interpolating when the interpolation problem $f(\lambda)=v_{\lambda}, \lambda \in \Lambda$, has a solution $f \in \hat{\mathcal{E}}^{\prime}(\mathbb{R})$ for every sequence of complex values $\left\{v_{\lambda}\right\}_{\lambda \in \Lambda}$ having the characteristic growth of $\hat{\mathcal{E}}^{\prime}(\mathbb{R})$ on $\Lambda$ (see the precise definition in Section 2).

The origin of the interest in $\hat{\mathcal{E}}^{\prime}(\mathbb{R})$-interpolation lies in its relationship with convolution equations and, in particular, with the density of exponential families $\left\{e^{i \lambda x}\right\}_{\lambda \in \Lambda}$ in the space of solutions $g \in \mathcal{E}(\mathbb{R})$ of equations of type $\mu \star g=0$, $\mu \in \mathcal{E}^{\prime}(\mathbb{R})$. Any solution $g$ to the convolution equation is the limit of linear combinations of $\left\{e^{i \lambda x}\right\}_{\lambda \in \Lambda}$ where $\Lambda$ is the zero set of $\hat{\mu}$. If, moreover, the sequence $\Lambda$ is $\hat{\mathcal{E}}^{\prime}(\mathbb{R})$-interpolating, then the series that represents $g$ enjoys better convergence properties. For more on this relationship see [EhMa74] or [BrGa95, Chapter 6] (in particular, Theorem 6.1.11).

The space $\hat{\mathcal{E}}^{\prime}(\mathbb{R})$ is a particular case of the algebras

$$
A_{p}=\{f \in H(\mathbb{C}): \log |f(z)| \leq A+B p(z) \text { for some } A, B>0\}
$$

associated to positive measurable weights $p$, obtained by taking

$$
p(z)=|\operatorname{Im} z|+\log \left(1+|z|^{2}\right) .
$$

Received by the editors May 17, 2004.

2000 Mathematics Subject Classification. Primary 30E05, 42A85.

Key words and phrases. Interpolating sequence, entire function.

The first and second authors were supported by DGICYT grant BFM2002-04072-C02-01 and CIRIT grant 2001-SGR00172. 
There exists an analytic characterization of interpolating sequences for general $A_{p}$ spaces when $p$ is subharmonic (see Theorem A below). However, a complete geometric description exists only for subharmonic weights $p$ which are both radial $(p(z)=p(|z|))$ and doubling $(p(2 z) \leq C p(z)$ for some $C>0)$; see BrLi95, Corollary 4.8].

For the weight (1) Ehrenpreis and Malliavin gave a necessary geometric condition which turns out to be sufficient provided that $\Lambda$ is a zero sequence of a slowly decreasing function (see EhMa74, Theorem 4] and its proof). Later Squires, probably unaware of Ehrenpreis and Malliavin's result (which was stated in terms of solutions to convolution equations), proved the same result [Sq83, Theorem 2].

In this paper we give a geometric characterization for $\hat{\mathcal{E}}^{\prime}(\mathbb{R})$-interpolating sequences (Theorem 1). The characterization shows in particular that the geometric condition given by Ehrenpreis \& Malliavin and Squires is also sufficient whenever the sequence is contained in the region

$$
|\operatorname{Im} z| \leq C \log \left(1+|z|^{2}\right) .
$$

In general, however, their condition alone is not sufficient.

A similar characterization is obtained for the more general Beurling weights. These weights appear naturally in the context of convolution equations when one replaces distributions with compact support with Beurling-Björck ultradistributions of compact support (see Bj66]). They are not necessarily subharmonic, but we will prove that they are equivalent to a subharmonic weight (see Lemma 8).

The paper is structured as follows. In Section 2 we give the precise definition of interpolating variety, discuss the background for the problem and state the main result. In Section 3 we prove that the geometric conditions of Theorem 1 are necessary, while in Section 4 we show that they are also sufficient.

A final remark about notation. $C$ will always denote a positive constant and its actual value may change from one occurrence to the next. $A=O(B)$ and $A \lesssim B$ mean that $A \leq c B$ for some $c>0$, and $A \simeq B$ is $A \lesssim B \lesssim A$.

\section{Preliminaries}

For the following definition and general background on the problem we refer to BrGa95, Chapter 2].

A measurable function $p: \mathbb{C} \longrightarrow \mathbb{R}_{+}$is called a weight if for some $C, D>0$ :

(a) $\log \left(1+|z|^{2}\right) \leq C p(z)$ for all $z \in \mathbb{C}$.

(b) $p(\zeta) \leq C p(z)+D$ if $|\zeta-z| \leq 1$.

The importance of these properties lies in their consequences for the ring $A_{p}$ defined in the introduction: (a) implies that $A_{p}$ contains all polynomials, and (b) that $A_{p}$ is closed under differentiation.

The algebra $A_{p}$ can be thought of as the union of the Hilbert spaces

$$
A_{p, \alpha}^{2}:=\left\{f \in H(\mathbb{C}):\|f\|_{A_{p, \alpha}^{2}}^{2}=\int_{\mathbb{C}}|f(z)|^{2} e^{-\alpha p(z)} d m(z)<\infty\right\}
$$

for $\alpha>0$, as well as the union of the Banach spaces

$$
A_{p, \alpha}^{\infty}:=\left\{f \in H(\mathbb{C}):\|f\|_{A_{p, \alpha}^{\infty}}=\sup _{z \in \mathbb{C}}|f(z)| e^{-\alpha p(z)}<\infty\right\} .
$$

Also, $A_{p}=\bigcup_{\alpha>0} A_{p, \alpha}^{\infty}$ has the structure of an (LF)-space with the topology of the inductive limit. 
Definition. Let $\Lambda$ be a discrete sequence in $\mathbb{C}$ and let $\left\{m_{\lambda}\right\}_{\lambda \in \Lambda}$ be a sequence of natural numbers. The pair $X=\left\{\left(\lambda, m_{\lambda}\right)\right\}_{\lambda \in \Lambda}$ is called an interpolating variety for the space $A_{p}$ if for every sequence of values $\left\{v_{\lambda}^{l}\right\}_{\lambda, l}, \lambda \in \Lambda, l=0, \ldots, m_{\lambda}-1$, with

$$
\sup _{\lambda \in \Lambda}\left(\sum_{l=0}^{m_{\lambda}-1}\left|v_{\lambda}^{l}\right|\right) e^{-\alpha p(\lambda)}<\infty
$$

for some $\alpha>0$, there exists $f \in A_{p}$ with

$$
\frac{f^{(l)}(\lambda)}{l !}=v_{\lambda}^{l}, \quad \lambda \in \Lambda ; \quad l=0, \ldots, m_{\lambda}-1 .
$$

The choice of condition (2) on the values to be interpolated reflects the fact that for every $f \in A_{p}$ there exists $\alpha>0$ such that

$$
\sup _{z \in \mathbb{C}}\left(\sum_{l=0}^{\infty}\left|\frac{f^{(l)}(z)}{l !}\right|\right) e^{-\alpha p(z)}<\infty .
$$

Thus, denoting by $A_{p}(X)$ the space of sequences $\left\{v_{\lambda}^{l}\right\}_{\lambda, l}$ satisfying (2) for some $\alpha>0$, we can equivalently define interpolating varieties $X$ as those such that the restriction operator

$$
\begin{aligned}
\mathcal{R}_{X}: A_{p}(\mathbb{C}) & \longrightarrow A_{p}(X) \\
f & \mapsto\left\{\frac{f^{(l)}(\lambda)}{l !}\right\}_{\lambda, l}
\end{aligned}
$$

is onto.

There exists an analytic characterization of $A_{p}$-interpolating varieties for general subharmonic weights $p$ (see [BrLi95, Corollary 3.5]). Results for $p(z)=|z|$ and the weight (11) were previously obtained respectively by Leont'ev Le72 and Squires Sq81.

Given a holomorphic function $f$ let $\mathcal{Z}(f)$ denote its zero variety, i.e., the set of pairs $\left(z, m_{z}\right) \in \mathbb{C} \times \mathbb{N}$ such that $f(z)=0$ with multiplicity $m_{z}$.

Theorem A. A variety $X=\left\{\left(\lambda, m_{\lambda}\right)\right\}_{\lambda \in \Lambda}$ is $A_{p}$-interpolating if and only if there exists $f \in A_{p}$ such that $X \subset \mathcal{Z}(f)$ and for some constants $\delta, C>0$,

$$
\left|\frac{f^{\left(m_{\lambda}\right)}(\lambda)}{m_{\lambda} !}\right| \geq \delta e^{-C p(\lambda)}, \quad \lambda \in \Lambda .
$$

We would like to give a geometric description of $A_{p}$-interpolating varieties for the non-isotropic Beurling weights

$$
p(z)=|\operatorname{Im} z|+\omega(|z|),
$$

where $\omega(t)$ is a subadditive increasing continuous function, normalized with $\omega(0)=$ 0 and such that:

(c) $\log (1+t) \lesssim \omega(t)$ for $t>1$.

(d) $\int_{0}^{\infty} \frac{\omega(t)}{1+t^{2}} d t<\infty$.

Canonical examples of such weights are given by $\omega(t)=\log \left(1+t^{2}\right)$ and $\omega(t)=t^{\gamma}$, $\gamma \in(0,1)$.

Beurling weights satisfy the following additional properties:

(e) For every $c>0$ there exists $C>0$ such that $p(\zeta) \leq C p(z)$ if $\zeta \in$ $D(z, c p(z))$. 
(f) For $\varepsilon>0$ small enough, there exists $C(\varepsilon)>0$ such that if $z \in D(\zeta, \varepsilon p(\zeta))$, then $p(\zeta) \leq C(\varepsilon) p(z)$. Also, $C(\varepsilon)$ tends to 1 as $\varepsilon$ goes to 0 .

(g) For $x \in \mathbb{R}^{+}$big enough, the function $\omega(x)$ does not oscillate too much. More precisely, for fixed $C>0$, if $y \in(x-C \omega(x), x+C \omega(x))$, then $1 / 2 \leq \omega(y) / \omega(x) \leq 2$ for $x$ big enough.

Properties (e) and (f) follow easily from the subadditivity of $\omega$. Property (g) follows from the subadditivity and the fact that $\omega(x)=o(|x| / \log |x|)(\operatorname{see}[\operatorname{Bj} 66$, Lemma 1.2.8]): for any $y \in(x-C \omega(x), x+C \omega(x))$,

$$
\begin{aligned}
\omega(x-C \omega(x)) & \leq \omega(y) \leq \omega(x+C \omega(x)) \leq \omega(x-C \omega(x))+\omega(2 C \omega(x)) \\
& \leq \omega(x-C \omega(x))+\omega(2 C x / \log x) \leq 2 \omega(x-C \omega(x)) .
\end{aligned}
$$

In order to state the geometric conditions on a variety $X$ as above, we consider the counting function $n(z, r)=\sum_{\lambda \in D(z, r)} m_{\lambda}$ and the integrated version

$$
N(z, r)=\int_{0}^{r} \frac{n(z, t)-n(z, 0)}{t} d t+n(z, 0) \log r .
$$

In case we want to specify the variety $X$ to which the functions $n$ and $N$ refer, we will use the notation $n(z, r, X)$ and $N(z, r, X)$ respectively.

We are ready to state our main result.

Theorem 1. A variety $X=\left\{\left(\lambda, m_{\lambda}\right)\right\}_{\lambda \in \Lambda}$ is $A_{p}$-interpolating if and only if:

(i) There is $C>0$ such that

$$
N(\lambda, p(\lambda), X) \leq C p(\lambda) \quad \forall \lambda \in \Lambda .
$$

(ii) The following Carleson-type condition holds:

$$
\sup _{x \in \mathbb{R}} \sum_{\lambda:|\operatorname{Im} \lambda|>\omega(|\lambda|)} m_{\lambda} \frac{|\operatorname{Im} \lambda|}{|x-\lambda|^{2}}<\infty .
$$

Since the Poisson kernel at $\lambda$ in the corresponding half-plane (upper half-plane if $\operatorname{Im} \lambda>0$ and lower half-plane when $\operatorname{Im} \lambda<0)$ is $P(\lambda, x)=\frac{|\operatorname{Im} \lambda|}{|x-\lambda|^{2}}$, a restatement of condition (ii) is that the measure $\sum_{\lambda:|\operatorname{Im} \lambda|>\omega(|\lambda|)} m_{\lambda} \delta_{\lambda}$ has bounded Poisson balayage.

Remark 2. Notice that for sequences $\Lambda$ within the region $|\operatorname{Im} z| \leq \omega(|z|)$, condition (i) (shown to be necessary by Ehrenpreis \& Malliavin and Squires) provides a complete characterization. However, this is not the case in general, i.e. condition (ii) does not follow from (i), as it is shown in the following example. Take the sequence $\Lambda$ contained in the sector $\mathcal{A}=\{z \in \mathbb{C} ;|\operatorname{Re} z|<\operatorname{Im} z\}$ and having in each segment $\left\{\operatorname{Im} z=2^{n}\right\} \cap \mathcal{A}$ exactly $2^{n}$ equispaced points. Then $\Lambda$ satisfies condition (i) (basically $n(\lambda, t) \leq t$ for $t \leq p(\lambda)$ ), but it does not satisfy (ii) (it is not even a Blaschke sequence).

\section{NECESSARY CONDITIONS}

A standard feature of the spaces $A_{p}$ is that the interpolation can be performed in a stable way. This consequence of the open mapping theorem for (LF)-spaces applied to the restriction mapping $\mathcal{R}_{X}$ defined in Section 2 (see BrGa95, Lemma 2.2.6]) is stated precisely in the following lemma. 
Lemma 3. If $X$ is an interpolating variety, there exist $C>0, M \in \mathbb{N}$ such that for every $\lambda \in \Lambda$ there are functions $f_{\lambda}, g_{\lambda} \in A_{p}$ with bounded norms $\left\|f_{\lambda}\right\|_{A_{p, M}^{\infty}},\left\|g_{\lambda}\right\|_{A_{p, M}^{\infty}}$ $\leq C$ and

$$
\begin{aligned}
& f_{\lambda}^{(l)}\left(\lambda^{\prime}\right) / l !=\delta_{\lambda \lambda^{\prime}} \delta_{l 0}, \\
& g_{\lambda}^{(l)}\left(\lambda^{\prime}\right) / l !=\delta_{\lambda \lambda^{\prime}} \delta_{l\left(m_{\lambda}-1\right)} \quad \forall \lambda, \lambda^{\prime} \in \Lambda, 0 \leq l \leq m_{\lambda} .
\end{aligned}
$$

An application of Jensen's Formula to the functions $f_{\lambda}, g_{\lambda}$ in the disk $D(\lambda, p(\lambda))$ gives the following result (see [EhMa74, Theorem 4] or [Sq83, Theorem 1]).

Theorem 4. If $X$ is $A_{p}$-interpolating, then condition (i) of Theorem 1 holds.

The necessity of condition (ii) is an immediate consequence of the following result. Assume that $\Lambda \cap \mathbb{R}=\emptyset$; otherwise move the horizontal line so that it does not touch any of the points in $\Lambda$. Let $\mathbb{H}$ denote the upper half-plane.

Proposition 5. Let $X$ be $A_{p}$-interpolating. There exist $C>0$ such that

$$
\sum_{\substack{\lambda^{\prime} \in \Lambda \cap \mathbb{H} \\ \lambda^{\prime} \neq \lambda}} m_{\lambda^{\prime}} \log \left|\frac{\lambda-\lambda^{\prime}}{\lambda-\bar{\lambda}^{\prime}}\right|^{-1} \leq C p(\lambda) \quad \text { for all } \lambda \in \Lambda \cap \mathbb{H} .
$$

Of course, an analogous result could be given for any upper $(\{z: \operatorname{Im} z>a\})$ or lower $(\{z: \operatorname{Im} z<a\})$ half-plane.

Before giving the proof of Proposition 5, we show that it implies condition (ii) of Theorem 1. Define $\Lambda_{+}=\Lambda \cap\{\operatorname{Im} z>\omega(|z|)\}$. Given $x \in \mathbb{R}$ consider $\lambda \in \Lambda_{+}$ such that $|x-\lambda|=\inf _{\Lambda_{+}}|x-\lambda|$. Then

$$
\left|\lambda-\bar{\lambda}^{\prime}\right| \leq|\lambda-x|+\left|x-\bar{\lambda}^{\prime}\right|=|\lambda-x|+\left|x-\lambda^{\prime}\right| \leq 2\left|x-\lambda^{\prime}\right|,
$$

and therefore

$$
\sum_{\lambda^{\prime} \in \Lambda_{+}} m_{\lambda^{\prime}} \frac{\left|\operatorname{Im} \lambda^{\prime}\right|}{\left|x-\bar{\lambda}^{\prime}\right|^{2}} \leq 2 \sum_{\lambda^{\prime} \in \Lambda_{+}} m_{\lambda^{\prime}} \frac{\left|\operatorname{Im} \lambda^{\prime}\right|}{\left|\lambda-\bar{\lambda}^{\prime}\right|^{2}} .
$$

The estimate $\log t^{-1} \geq 1-t$ for $t \in(0,1)$ shows that

$$
\sum_{\substack{\lambda^{\prime} \in \Lambda_{+} \\ \lambda^{\prime} \neq \lambda}} m_{\lambda^{\prime}} \frac{|\operatorname{Im} \lambda|\left|\operatorname{Im} \lambda^{\prime}\right|}{\left|\lambda-\bar{\lambda}^{\prime}\right|^{2}} \leq \sum_{\substack{\lambda^{\prime} \in \Lambda_{+} \\ \lambda^{\prime} \neq \lambda}} m_{\lambda^{\prime}} \log \left|\frac{\lambda-\lambda^{\prime}}{\lambda-\bar{\lambda}^{\prime}}\right|^{-1} .
$$

Since $p(\lambda) \simeq|\operatorname{Im} \lambda|$ for $\lambda \in \Lambda_{+}$, it is clear that this implies condition (ii) of Theorem 1

Remark 6. The necessary condition of Proposition 5 can be seen as a Carleson-type condition; it can be rewritten as

$$
\left|B_{\lambda}(\lambda)\right| \geq \delta e^{-C p(\lambda)}, \quad \lambda \in \Lambda \cap \mathbb{H}
$$

where $B$ denotes the Blaschke product in $\mathbb{H}$ of $\left\{\left(\lambda, m_{\lambda}\right)\right\}_{\lambda \in \Lambda \cap \mathbb{H}}$, and

$$
B_{\lambda}(z)=B(z)\left(\frac{z-\bar{\lambda}}{z-\lambda}\right)^{m_{\lambda}} .
$$

It can also be seen as density conditions for the counting function associated to the hyperbolic metric in the half-plane. Letting $\nu=\sum_{\lambda \in \Lambda \cap \mathbb{H}} m_{\lambda} \delta_{\lambda}$ and using the distribution function we have

$$
\sum_{\lambda \in \Lambda \cap \mathbb{H}} m_{\lambda} \log \left|\frac{z-\lambda}{z-\bar{\lambda}}\right|^{-1}=\int_{\mathbb{H}} \log \left|\frac{z-\zeta}{z-\bar{\zeta}}\right|^{-1} d \nu(\zeta)=\int_{0}^{1} \frac{n_{\mathbb{H}}(z, t)}{t} d t
$$


where

$$
D_{\mathbb{H}}(z, t)=\left\{\zeta:\left|\frac{z-\zeta}{z-\bar{\zeta}}\right|<t\right\}, \quad \text { and } \quad n_{\mathbb{H}}(z, t):=\nu\left(D_{\mathbb{H}}(z, t)\right)
$$

is the number of points of $\Lambda$ in the pseudohyperbolic disk of "center" $z$ and "radius" $t$ (actually the true disk of center $\operatorname{Re} z+i \frac{1+t^{2}}{1-t^{2}} \operatorname{Im} z$ and radius $\frac{2 t}{1-t^{2}} \operatorname{Im} z$ ).

Proof of Proposition 5. Let $z=x+i y$ and consider the Poisson transform of $\omega(|t|)$ :

$$
u(z):=P[\omega](z)=\int_{\mathbb{R}} \frac{y \omega(|t|)}{(x-t)^{2}+y^{2}} d t,
$$

which converges by $(\mathrm{d})$. Define $H=\exp (u+i \tilde{u})$, where $\tilde{u}$ is a harmonic conjugate of $u$.

Given $\lambda \in \Lambda \cap \mathbb{H}$, take the function $f_{\lambda}$ given by Lemma 3 and define

$$
h_{\lambda}(z)=\frac{f_{\lambda}(z) e^{i M_{1} z}}{(H(z))^{M_{2}}}
$$

with $M_{1}, M_{2}$ to be chosen. It is clear that $h_{\lambda}$ is holomorphic in $\mathbb{H}$. On the other hand, for all $z$ in the upper half-plane $|\log | H(z)|-\omega(|\operatorname{Re} z|)| \leq A+B|\operatorname{Im} z|$; see Bj66, Lemma 1.3.11]. Moreover, $|\omega(|\operatorname{Re} z|)-\omega(|z|)| \leq \omega(|\operatorname{Im} z|) \leq A+B|\operatorname{Im} z|$, thus $|\log | H(z)|-\omega(|z|)| \leq A+B|\operatorname{Im} z|$. Therefore, if $M_{1}$ and $M_{2}$ are big enough, $h_{\lambda}$ is bounded in $\mathbb{H}$ by a constant which does not depend on $\lambda$ :

$$
\left|h_{\lambda}(z)\right| \leq C e^{M p(z)-M_{1} \operatorname{Im} z-M_{2} \log |H(z)|} \lesssim 1 .
$$

Also,

$$
\left|h_{\lambda}(\lambda)\right|=e^{-M_{1} \operatorname{Im} \lambda-M_{2} \log |H(\lambda)|} \geq e^{-C p(\lambda)} .
$$

Now apply Jensen's formula in the half-plane to the function $h_{\lambda}$ :

$$
\log \left|h_{\lambda}(\lambda)\right|=\int_{\mathbb{R}} P(\lambda, x) \log \left|h_{\lambda}(x)\right| d x-\int_{\mathbb{H}} G(\lambda, \zeta) \Delta \log \left|h_{\lambda}(\zeta)\right|,
$$

where $P(\lambda, x)$ denotes the Poisson kernel and $G(\lambda, \zeta)=\log \left|\frac{\lambda-\zeta}{\lambda-\bar{\zeta}}\right|^{-1}$ is the Green function in $\mathbb{H}$ with pole in $\lambda$.

Since $h_{\lambda}$ vanishes on $\Lambda \backslash\{\lambda\}$, Jensen's Formula and the estimates above yield

$$
\sum_{\substack{\lambda^{\prime} \in \Lambda \cap \mathbb{H} \\ \lambda^{\prime} \neq \lambda}} m_{\lambda^{\prime}} \log \left|\frac{\lambda-\lambda^{\prime}}{\lambda-\bar{\lambda}^{\prime}}\right|^{-1} \leq \sup _{\mathbb{R}} \log \left|h_{\lambda}\right|-\log \left|h_{\lambda}(\lambda)\right| \lesssim p(\lambda) .
$$

\section{Sufficient COnditions}

We split the sequence into three pieces, according to the non-isotropy of the weight $p$. Consider the regions

$$
\begin{aligned}
\Omega_{0} & =\{z \in \mathbb{C}:|\operatorname{Im} z| \leq \omega(|z|)\}, \\
\Omega_{+} & =\{z \in \mathbb{C}: \operatorname{Im} z>\omega(|z|)\}, \\
\Omega_{-} & =\{z \in \mathbb{C}: \operatorname{Im} z<-\omega(|z|)\},
\end{aligned}
$$

and define $\Lambda_{0}=\Lambda \cap \Omega_{0}, \Lambda_{+}=\Lambda \cap \Omega_{+}$and $\Lambda_{-}=\Lambda \cap \Omega_{-}$. Let also $X_{0}=$ $\left\{\left(\lambda, m_{\lambda}\right)\right\}_{\lambda \in \Lambda_{0}}, X_{+}=\left\{\left(\lambda, m_{\lambda}\right)\right\}_{\lambda \in \Lambda_{+}}$and $X_{-}=\left\{\left(\lambda, m_{\lambda}\right)\right\}_{\lambda \in \Lambda_{-}}$.

It is enough to prove that each piece $X_{+}, X_{-}, X_{0}$ of the variety $X$ is $A_{p}$-interpolating. This is so because $X$ is weakly separated (see Lemma 7 (i) below), and 
a weakly separated union of a finite number of $A_{p}$-interpolating varieties is also $A_{p}$-interpolating [Ou03, Theorem II.1]. It is also clear that the varieties $X^{+}$and $X^{-}$can be dealt with similarly.

We start with some easy consequences of condition (i) of Theorem 1

Lemma 7. If condition (i) in Theorem 1 holds, then

(i) $X$ is weakly separated: there exist $\delta, C>0$ such that the disks $D_{\lambda}=$ $D\left(\lambda, \delta e^{-C \frac{p(\lambda)}{m_{\lambda}}}\right)$ are pairwise disjoint, i.e.

$$
\left|\lambda-\lambda^{\prime}\right| \geq 2 \delta \max \left[e^{-C \frac{p(\lambda)}{m_{\lambda}}}, e^{-C \frac{p\left(\lambda^{\prime}\right)}{m_{\lambda^{\prime}}}}\right], \quad \lambda \neq \lambda^{\prime} .
$$

(ii) There exist $\varepsilon, C>0$ such that $n(z, \varepsilon p(z), X) \leq C p(z), \forall z \in \mathbb{C}$.

Proof. (i) If there exists $\lambda^{\prime}$ such that $\left|\lambda^{\prime}-\lambda\right|<1$, then

$$
N(\lambda, p(\lambda), X) \geq \int_{\left|\lambda^{\prime}-\lambda\right|}^{1} \frac{n(\lambda, t)-m_{\lambda}}{t} d t \geq \int_{\left|\lambda^{\prime}-\lambda\right|}^{1} \frac{m_{\lambda^{\prime}}}{t} d t=\log \left(\frac{1}{\left|\lambda^{\prime}-\lambda\right|}\right)^{m_{\lambda^{\prime}}} .
$$

Using condition (i) in Theorem 1 and reversing the roles of $\lambda$ and $\lambda^{\prime}$ we obtain the desired estimate.

(ii) When $z=\lambda \in \Lambda$, this is immediate from the estimate

$$
\int_{1 / 2 p(\lambda)}^{p(\lambda)} \frac{n(\lambda, 1 / 2 p(\lambda))-1}{t} d t \leq N(\lambda, p(\lambda)) .
$$

When $z \notin \Lambda$, then let $\varepsilon>0$ be such that $\zeta \in D(z, \varepsilon p(z))$ implies

$$
D(z, \varepsilon p(z)) \subset D(\zeta, 1 / 2 p(\zeta)),
$$

which exists by property (f) of the weight. Take $\lambda \in D(z, \varepsilon p(z)$ ) (if there is no such $\lambda$ the estimate is obviously true). Then, by the previous case and property (e) of the weight

$$
n(z, \varepsilon p(z)) \leq n(\lambda, 1 / 2 p(\lambda)) \lesssim p(\lambda) \lesssim p(z)
$$

4.1. Case $\Lambda_{0}$. We would like to prove that $X_{0}=\left\{\left(\lambda, m_{\lambda}\right)\right\}_{\lambda \in \Lambda_{0}}$ is $A_{p}$-interpolating using a $\bar{\partial}$-scheme. This is easier if we can regularize the weight in the following way.

Lemma 8. There exists $\tilde{p}$ subharmonic in $\mathbb{C}$ such that $p(z) \simeq \tilde{p}(z)$ and

$$
1 / \tilde{p}(z) \lesssim \Delta \tilde{p}(z) \quad \text { if } \quad|\operatorname{Im} z| \leq 2 \omega(|z|) .
$$

The fact that $p \simeq \tilde{p}$ clearly implies that $A_{p}=A_{\tilde{p}}$ and the interpolating varieties for $A_{p}$ and $A_{\tilde{p}}$ are the same.

Proof. We will construct $\tilde{p}(z)=|\operatorname{Im} z|+r(z)$, where $r$ satisfies the following properties:

(i) $r \geq 0$ and $\tilde{p}$ is subharmonic in $\mathbb{C}$.

(ii) $r(z)=0$ if $|\operatorname{Im} z| \geq 10 \omega(|z|)$.

(iii) $1 / p(z) \lesssim \Delta \tilde{p}(z)$ and $r(z) \simeq \omega(|z|)$ if $|\operatorname{Im} z| \leq 2 \omega(|z|)$. 
In order to construct $r$, we partition the real line into intervals $I_{n}$ defined in the following way.

Let $x_{1}>1, x_{n+1}=x_{n}+\omega\left(x_{n}\right)$ for $n \geq 1$ and $x_{n}=-x_{-n}$ for $n \leq-1$. Set $I_{0}=\left[x_{-1}, x_{1}\right], I_{n}=\left[x_{n}, x_{n+1}\right]$ for $n \geq 1$ and $I_{n}=\left[x_{n-1}, x_{n}\right]$ for $n \leq-1$. Denote by $\omega_{n}$ the length of $I_{n}$.

We consider two measures in $\mathbb{C}$. The first one is the usual length measure $d \nu$ in $\mathbb{R}$, which we split $d \nu=\sum_{n} d \nu_{n}$, with $d \nu_{n}=d x_{\mid I_{n}}$. The second one is defined as a sum of convolutions of the $d \nu_{n}$ 's: let

$$
d \mu_{n}(z)=\left(\frac{1}{100 \pi \omega_{n}^{2}} \int_{I_{n}} \chi_{D_{n}}(z-x) d x\right) d m(z),
$$

where $D_{n}=D\left(0,10 \omega_{n}\right)$, and define $d \mu=\sum_{n} d \mu_{n}$.

Notice that when $z$ is at a distance of $I_{n}$ smaller than $2 \omega_{n}$, we can use property (g) of the Beurling weights to deduce that $d \mu(z) \simeq 1 / \omega(|z|) \simeq 1 / p(z)$. Hence $d \mu(z) \simeq d m(z) / p(z)$.

Define

$$
r(z)=\int_{\mathbb{C}} \log |z-w|(d \mu(w)-d \nu(w)) .
$$

Since $\Delta|\operatorname{Im} z|=d \nu$, we have $\Delta \tilde{p}=d \mu \geq 0$.

Let $S_{n}$ denote the support of $\mu_{n}$. Let

$r_{n}(z):=\int_{\mathbb{C}} \log |z-w|\left(d \mu_{n}(w)-d \nu_{n}(w)\right)=\int_{S_{n}} \log |z-w| d \mu_{n}(w)-\int_{I_{n}} \log |z-x| d x$.

Using the definition of $\mu_{n}$ and reversing the order of integration we get

$$
r_{n}(z)=\int_{I_{n}} M(x) d x
$$

where

$$
M(x)=\frac{1}{100 \pi \omega_{n}^{2}} \int_{D\left(x, 10 \omega_{n}\right)} \log |z-w| d m(w)-\log |z-x| \geq 0 .
$$

In particular, $r$ is non-negative in $\mathbb{C}$.

If $z \notin S_{n}$ and $x \in I_{n}, \log |z-w|$ is harmonic in $D\left(x, 10 \omega_{n}\right)$, hence $r_{n}(z)=0$.

Suppose now $z \in D\left(x_{n}, 3 \omega_{n}\right)$. Then, for each $x \in I_{n},|z-x| \leq 4 \omega_{n}$ and

$$
M(x) \geq \frac{1}{100 \pi \omega_{n}^{2}} \int_{9 \omega_{n} \leq|w-x| \leq 10 \omega_{n}} \log \frac{|z-w|}{|z-x|} d m(w) \gtrsim 1 .
$$

Thus, $r_{n}(z) \gtrsim \omega_{n} \gtrsim \omega(|z|)$.

If $z \in S_{n}$, using that $\mu_{n}$ and $\nu_{n}$ have the same mass $\omega\left(x_{n}\right)$, we obtain

$$
\begin{gathered}
\int_{\mathbb{C}} \log |z-w|\left(d \mu_{n}(w)-d \nu_{n}(w)\right) \leq \int_{\mathbb{C}}\left|\log \frac{|z-w|}{\omega\left(x_{n}\right)}\right|\left(d \mu_{n}(w)+d \nu_{n}(w)\right) \\
\lesssim \int_{\mathbb{C}}\left|\log \frac{\left|x_{n}-w\right|}{\omega\left(x_{n}\right)}\right|\left(d \mu_{n}(w)+d \nu_{n}(w)\right) \lesssim \omega(|z|) .
\end{gathered}
$$

Since $|\operatorname{Im} z| \leq 2 \omega(|z|), z$ belongs at most to a finite number of $S_{n}$ 's and at least to one $D\left(x_{n}, 10 \omega_{n}\right)$, by property ( $\mathrm{g}$ ) of the Beurling weights, we are done.

Let us prove now that $X_{0}$ is $A_{\tilde{p}}$-interpolating. In view of Lemma 8 , we assume that $1 / p \lesssim \Delta p$ on $|\operatorname{Im} z| \leq 2 \omega(|z|)$.

Consider the separation radii $\delta_{\lambda}:=\delta e^{-C p(\lambda)}$ given by Lemma 7 (i). 
Given a sequence of values $\left\{v_{\lambda}^{\ell}\right\}_{\lambda, \ell}$ satisfying (2), define the smooth interpolating function

$$
F(z)=\sum_{\lambda \in \Lambda_{0}} p_{\lambda}(z) \mathcal{X}\left(\frac{|z-\lambda|^{2}}{\delta_{\lambda}^{2}}\right)
$$

where $p_{\lambda}(z)=\sum_{l=0}^{m_{\lambda}-1} v_{\lambda}^{l}(z-\lambda)^{l}$ and $\mathcal{X}$ is a smooth cut-off function with $\left|\mathcal{X}^{\prime}\right| \lesssim 1$, $\mathcal{X}(x)=1$ if $|x| \leq 1$ and $\mathcal{X}(x)=0$ if $|x| \geq 2$.

It is clear that $F^{(l)}(\lambda) / l !=v_{\lambda}^{l}$, and that $F$ has the characteristic growth of $A_{p}$ functions; the support of $F$ is contained in $\bigcup_{\lambda} D_{\lambda}$ and for $z \in D_{\lambda}$,

$$
|F(z)| \leq \sum_{l=0}^{m_{\lambda}-1}\left|v_{\lambda}^{l}\right| \leq C e^{\alpha p(\lambda)} \lesssim e^{K p(z)}
$$

There is also a good estimate on $\bar{\partial} F$. Its support is the union of the annuli

$$
C_{\lambda}=\left\{z \in \mathbb{C}: \delta_{\lambda} \leq|z-\lambda| \leq 2 \delta_{\lambda}\right\}
$$

and for $z \in C_{\lambda}$,

$$
\left|\frac{\partial F}{\partial \bar{z}}(z)\right| \lesssim \sum_{l=0}^{m_{\lambda}-1}\left|v_{\lambda}^{l} \| \mathcal{X}^{\prime}\right| \frac{1}{\delta_{\lambda}} \lesssim e^{C p(\lambda)} \lesssim e^{K p(z)},
$$

for $K$ big enough.

Altogether, there exists $\gamma>0$ such that

$$
\int_{\mathbb{C}}|F(z)|^{2} e^{-\gamma p(z)}<\infty, \quad \int_{\mathbb{C}}|\bar{\partial} F(z)|^{2} e^{-\gamma p(z)}<\infty .
$$

Now, when looking for a holomorphic interpolating function of the form $f=F-u$, we are led to the $\bar{\partial}$-problem

$$
\bar{\partial} u=\bar{\partial} F
$$

which we solve using Hörmander's theorem [Ho94, Theorem 4.2.1]: given a (pluri)subharmonic function $\psi$ in $\mathbb{C}$, there exists a solution $u$ to the above equation such that

We apply Hörmander's theorem with

$$
2 \int_{\mathbb{C}}|u|^{2} \frac{e^{-\psi}}{\left(1+|z|^{2}\right)^{2}} d m \leq \int_{\mathbb{C}}|\bar{\partial} F|^{2} e^{-\psi} d m .
$$

$$
\psi_{\beta}(z)=\beta p(z)+v(z)
$$

where $\beta>0$ will be chosen later on and

$$
v(z)=\sum_{\lambda \in \Lambda_{0}} m_{\lambda}\left[\log |z-\lambda|^{2}-\frac{1}{\pi \varepsilon^{2} p^{2}(\lambda)} \int_{D(\lambda, \varepsilon p(\lambda))} \log |z-\zeta|^{2} d m(\zeta)\right] .
$$

Here $\varepsilon$ is a fixed small constant to be determined later on.

Integrating by parts the equality

$$
\int_{0}^{2 \pi} \log \left|a-r e^{i \theta}\right|^{2} \frac{d \theta}{2 \pi}=\left\{\begin{array}{lll}
\log |a|^{2} & \text { if } & |a|>r \\
\log r^{2} & \text { if } & |a| \leq r
\end{array}\right.
$$

one sees that for $a \in \mathbb{C}$ and $r>0$,

$$
\log |a|^{2}-\frac{1}{\pi r^{2}} \int_{D(a, r)} \log |\zeta|^{2} d m(\zeta)= \begin{cases}\log \left|\frac{a}{r}\right|^{2}+1-\left|\frac{a}{r}\right|^{2} & \text { if } \quad|a| \leq r \\ 0 & \text { if } \quad|a|>r\end{cases}
$$


Thus

$$
v(z)=\sum_{\lambda:|\lambda-z| \leq \varepsilon p(\lambda)} m_{\lambda}\left[\log \frac{|z-\lambda|^{2}}{\varepsilon^{2} p^{2}(\lambda)}+1-\frac{|z-\lambda|^{2}}{\varepsilon^{2} p^{2}(\lambda)}\right] .
$$

In particular, $v \leq 0$ and $\Delta v(z)=0$ if $z \notin \bigcup_{\lambda} D(\lambda, \varepsilon p(\lambda))$. For $z \in \bigcup_{\lambda} D(\lambda, \varepsilon p(\lambda))$ we have $|\operatorname{Im} z| \leq 2 \omega(|z|)$ and

$$
\Delta v(z) \geq \sum_{\lambda:|\lambda-z| \leq \varepsilon p(\lambda)} \frac{-m_{\lambda}}{\varepsilon^{2} p^{2}(\lambda)} \gtrsim \sum_{\lambda:|\lambda-z| \leq C(\varepsilon) p(z)} \frac{-m_{\lambda}}{p^{2}(z)}=-\frac{n(z, C(\varepsilon) p(z))}{p^{2}(z)} .
$$

As observed in Lemma 7 (ii), with $\varepsilon$ small enough $n(z, C(\varepsilon) p(z)) \lesssim p(z)$, thus $\Delta v(z) \gtrsim-1 / p(z)$. This and (3) show that $\psi_{\beta}$ is subharmonic if $\beta$ is chosen big enough.

Also, we deduce from (c) that for any $\beta^{\prime}>\beta$,

$$
\int_{\mathbb{C}}|u|^{2} e^{-\beta^{\prime} p} d m \lesssim \int_{\mathbb{C}}|u|^{2} \frac{e^{-\psi_{\beta}}}{\left(1+|z|^{2}\right)^{2}} d m \lesssim \int_{\mathbb{C}}|\bar{\partial} F|^{2} e^{-\psi_{\beta}} d m
$$

We need to control $\psi_{\beta}$ on the support of $\bar{\partial} F$. For $z \in C_{\lambda}$,

$$
\begin{aligned}
& \left|\psi_{\beta}(z)-\beta p(z)\right| \leq \sum_{\lambda:|\lambda-z| \leq \varepsilon p(\lambda)} m_{\lambda} \log \frac{\varepsilon^{2} p^{2}(\lambda)}{|z-\lambda|^{2}} \\
& \simeq m_{\lambda} \log \frac{\varepsilon^{2} p^{2}(\lambda)}{|z-\lambda|^{2}}+\sum_{\substack{\lambda^{\prime}:\left|z-\lambda^{\prime}\right| \leq \varepsilon p\left(\lambda^{\prime}\right) \\
\lambda^{\prime} \neq \lambda}} m_{\lambda^{\prime}} \log \frac{\varepsilon^{2} p^{2}\left(\lambda^{\prime}\right)}{\left|z-\lambda^{\prime}\right|^{2}} \\
& \lesssim p(\lambda)+\sum_{\substack{\lambda^{\prime}:\left|\lambda^{\prime}-z\right| \leq C(\varepsilon) p(z) \\
\lambda^{\prime} \neq \lambda}} m_{\lambda^{\prime}} \log \frac{C(\varepsilon)^{2} p^{2}(z)}{\left|z-\lambda^{\prime}\right|^{2}} \\
& \lesssim p(z)+N(z, C(\varepsilon) p(z)) .
\end{aligned}
$$

Claim 9. For $\varepsilon$ small enough $N(z, C(\varepsilon) p(z)) \lesssim p(z)$ for all $z \in \operatorname{supp}(\bar{\partial} F)$.

Assuming the claim we have $\left|\psi_{\beta}(z)-\beta p(z)\right| \leq K p(z)$ on $\operatorname{supp}(\bar{\partial} F)$. Therefore, for $\beta$ big enough

$$
\int_{\mathbb{C}}|u|^{2} e^{-\beta^{\prime} p} d m \lesssim \int_{\mathbb{C}}|\bar{\partial} F|^{2} e^{-\psi_{\beta}} d m \leq \int_{\mathbb{C}}|\bar{\partial} F|^{2} e^{-\gamma p} d m<\infty .
$$

This shows that $f:=F-u \in A_{p}$. Since $e^{-\psi_{\beta}} \simeq|z-\lambda|^{-2 m_{\lambda}}$ around each $\lambda$, also $u^{(l)}(\lambda)=0$ for all $\lambda \in \Lambda, l=0, \ldots, m_{\lambda}-1$, and therefore $f^{(l)}(\lambda) / l !=F^{(l)}(\lambda) / l !=$ $v_{\lambda}^{l}$, as required.

Proof of the claim. Assume $z \in C_{\lambda}$ and observe that $n(z, t)=0$ for $t<\delta_{\lambda}$ and that $n(z, t) \leq m_{\lambda}$ for $\delta_{\lambda} \leq t<2 \delta_{\lambda}$. Since $D(z, t) \subset D\left(\lambda, t+2 \delta_{\lambda}\right)$ and $|z|<|\lambda|+2 \delta_{\lambda}$, we have (changing into $s=t+2 \delta_{\lambda}$ )

$$
\begin{aligned}
N(z, C(\varepsilon) p(z)) & \leq \int_{\delta_{\lambda}}^{2 \delta_{\lambda}} \frac{m_{\lambda}}{t} d t+\int_{2 \delta_{\lambda}}^{C(\varepsilon) p(z)} \frac{n(z, t)-m_{\lambda}}{t} d t \\
& \leq p(\lambda)+\int_{4 \delta_{\lambda}}^{C(\varepsilon) p(z)+2 \delta_{\lambda}} \frac{n(\lambda, s)-m_{\lambda}}{s-2 \delta_{\lambda}} d s \\
& \lesssim p(\lambda)+\int_{4 \delta_{\lambda}}^{C(\varepsilon) p(z)+2 \delta_{\lambda}} \frac{n(\lambda, s)-m_{\lambda}}{s / 2} d s \lesssim p(\lambda)+N\left(\lambda, C^{\prime}(\varepsilon) p(\lambda)\right) .
\end{aligned}
$$


From the properties of the weight and the hypothesis we have finally that for $\varepsilon$ small $N(z, C(\varepsilon) p(z)) \lesssim p(\lambda) \lesssim p(z)$.

4.2. Case $\Lambda^{+}$. According to Theorem A, it is enough to construct a function $G \in$ $A_{p}$ such that $X_{+} \subset \mathcal{Z}(G)$ and

$$
\frac{\left|G^{\left(m_{\lambda}\right)}(\lambda)\right|}{m_{\lambda} !} \geq \varepsilon e^{-K p(\lambda)}, \quad \lambda \in \Lambda_{+},
$$

for some constants $\varepsilon, k>0$. In fact, the hypotheses of Theorem A require the weight $p$ to be subharmonic, and our weights are not necessarily so. Nevertheless, by Lemma 8 , there exists a subharmonic weight $\tilde{p}$ equivalent to $p$, and we may apply Theorem A to $\tilde{p}$.

Take any entire function $F$ such that $\mathcal{Z}(F)=X_{+}$. Since the necessary conditions imply that $X_{+}$satisfies the Blaschke condition in $\mathbb{H}$, we can consider also the Blaschke product

$$
B(z)=\prod_{\lambda \in \Lambda_{+}}\left(\frac{z-\lambda}{z-\bar{\lambda}}\right)^{m_{\lambda}}, \quad z \in \mathbb{H}
$$

Define

$$
\phi(z)= \begin{cases}\log \left|\frac{F(z)}{B(z)}\right|, & \operatorname{Im} z>0, \\ \log |F(z)|, & \operatorname{Im} z \leq 0 .\end{cases}
$$

Lemma 10. $\phi$ is harmonic outside the real axis, subharmonic on $\mathbb{C}$ and its Laplacian is uniformly bounded.

Proof. It is clear, by definition, that $\phi$ is harmonic on $\mathbb{C} \backslash \mathbb{R}$. In order to prove that $\phi$ is subharmonic on $\mathbb{C}$, it is enough to check the mean inequality for $x \in \mathbb{R}$. We have

$$
\phi(x)=\log |F(x)| \leq \frac{1}{2 \pi} \int_{0}^{2 \pi} \log \left|F\left(x+r e^{i \theta}\right)\right| d \theta \leq \frac{1}{2 \pi} \int_{0}^{2 \pi} \phi\left(x+r e^{i \theta}\right) d \theta .
$$

Since $\Delta \log |F| \equiv 0$ around $\mathbb{R}$, it is enough to compute the Laplacian of

$$
\psi(z)=\left\{\begin{array}{cc}
\log \frac{1}{|B(z)|}, & \operatorname{Im} z>0 \\
0, & \operatorname{Im} z \leq 0
\end{array}\right.
$$

Being

$$
\log \frac{1}{|B(z)|}=\frac{1}{2} \sum_{\lambda \in \Lambda^{+}} m_{\lambda} \log \left|\frac{z-\bar{\lambda}}{z-\lambda}\right|^{2}
$$

it will be enough to compute the Laplacian of each term

$$
\psi_{\lambda}(z)=\left\{\begin{array}{cc}
\log \left|\frac{z-\bar{\lambda}}{z-\lambda}\right|^{2}, & \operatorname{Im} z>0, \\
0, & \operatorname{Im} z \leq 0 .
\end{array}\right.
$$

It is clear that $\partial \psi_{\lambda} / \partial x=0$ on $\mathbb{R}$, hence $\Delta \psi_{\lambda}=\partial^{2} \psi_{\lambda} / \partial y^{2}$. Since $\psi_{\lambda}$ is continuous around $\mathbb{R}$, this Laplacian has a magnitude equivalent to the jump of the first derivative of $\psi_{\lambda}$. The derivative of the Green function on the half-plane with respect to the normal direction $y$ is the Poisson kernel:

$$
\frac{\partial}{\partial y} \log \left|\frac{z-\bar{\lambda}}{z-\lambda}\right|_{\mid y=0}^{2}=\frac{4 \operatorname{Im} \lambda}{|x-\lambda|^{2}}
$$


Therefore,

$$
\Delta \phi(x)=4 \sum_{\lambda \in \Lambda^{+}} m_{\lambda} \frac{\operatorname{Im} \lambda}{|x-\lambda|^{2}} d x
$$

which is bounded by the hypothesis.

Define

$$
\Psi(z)=N|\operatorname{Im} z|-\phi(z) .
$$

Observe that $\Delta \Psi(z)=N d x-\Delta \phi(x) d x$, thus according to the previous lemma $\Delta \Psi \simeq d x$ when $N \in \mathbb{N}$ is big enough. In this situation, according to OrSe99, Lemma 3], there exists a multiplier associated to $\Psi$, i.e., an entire function $h$ such that:

(a) $\mathcal{Z}(h)$ is a separated sequence contained in $\mathbb{R}$.

(b) Given any $\varepsilon>0,|h(z)| \simeq \exp (\Psi(z))$ for all points $z$ such that $d(z, \mathcal{Z}(h))$ $>\varepsilon$.

Define now $G=h F$. It is clear that $G \in A_{p}$ :

$$
|G(z)| \lesssim e^{\Psi(z)+\log |F(z)|} \leq e^{\Psi(z)+\phi(z)} \leq e^{N p(z)}, \quad z \in \mathbb{C} .
$$

It is also clear that $X_{+} \subset \mathcal{Z}(G)$, since $X_{+} \subset \mathcal{Z}(F)$.

In order to prove that there exist $\varepsilon, C>0$ such that

$$
\left|\frac{G^{\left(m_{\lambda}\right)}(\lambda)}{m_{\lambda} !}\right| \geq \varepsilon e^{-C p(\lambda)}
$$

consider then the disjoint disks $D_{\lambda}=D\left(\lambda, \delta_{\lambda}\right), \delta_{\lambda}=\delta e^{-C \frac{p(\lambda)}{m_{\lambda}}}$ given by Lemmal 7 (i). Since $\Lambda_{+}$is far from $\mathcal{Z}(h)$, the estimate

$$
|G(z)|=|h(z)| e^{\phi(z)}|B(z)| \simeq e^{N|\operatorname{Im} z|}|B(z)|, \quad z \in \partial D_{\lambda},
$$

holds.

Claim 11. There exists $C>0$ such that $|B(z)| \geq \epsilon e^{-C p(z)}, z \in \partial D_{\lambda}$.

Assuming this we have $|G(z)| \gtrsim e^{-C p(z)}$ for all $z \in \partial D_{\lambda}$. Define then $g(z)=$ $G(z) /(z-\lambda)^{m_{\lambda}}$. It is clear that $g$ is holomorphic, non-vanishing in $D_{\lambda}$, and $|g(z)| \gtrsim$ $e^{-c p(\lambda)}$ for $z \in \partial D_{\lambda}$. By the minimum principle

$$
\left|\frac{G^{\left(m_{\lambda}\right)}(\lambda)}{m_{\lambda} !}\right|=|g(0)| \gtrsim e^{-c p(\lambda)}
$$

as desired.

Proof of the claim. As observed in Remark 6(b), the estimate we want to prove is equivalent to

$$
\int_{0}^{1} \frac{n_{\mathbb{H}}(z, t)}{t} d t \lesssim p(z), \quad z \in \partial D_{\lambda}
$$

This is proved like Claim 9, except we replace the Euclidean disks by the hyperbolic ones. We have

$$
\int_{0}^{1} \frac{n_{\mathbb{H}}(z, t)}{t} d t \lesssim \int_{\delta_{\lambda}}^{2 \delta_{\lambda}} \frac{m_{\lambda}}{t} d t+\int_{2 \delta_{\lambda}}^{1} \frac{n_{\mathbb{H}}(z, t)-m_{\lambda}}{t} d t .
$$


The first term is controlled by $p(\lambda)$. In order to control the second term observe that $D_{\mathbb{H}}(z, t) \subset D_{\mathbb{H}}\left(\lambda, \frac{t+\delta_{\lambda}}{1+t \delta_{\lambda}}\right)$; hence changing the variable into $s=\frac{t+\delta_{\lambda}}{1+t \delta_{\lambda}}$ we get

$$
\int_{2 \delta_{\lambda}}^{1} \frac{n_{\mathbb{H}}(z, t)-m_{\lambda}}{t} d t \leq \int_{\frac{3 \delta_{\lambda}}{1+2 \delta_{\lambda}^{2}}}^{1} \frac{n_{\mathbb{H}}(\lambda, s)-m_{\lambda}}{s-\delta_{\lambda}} \frac{1-\delta_{\lambda}^{2}}{\left(1-\delta_{\lambda}\right)^{2}} d s .
$$

There is no restriction in assuming that $\delta_{\lambda}<1 / 2$. Then $\frac{3 \delta_{\lambda}}{1+2 \delta_{\lambda}^{2}}>2 \delta_{\lambda}$ and therefore $s-\delta_{\lambda}>s / 2$. With this and condition (ii) in Theorem 1 we obtain

$$
\int_{0}^{1} \frac{n_{\mathbb{H}}(z, t)}{t} d t \lesssim p(\lambda)+\int_{0}^{1} \frac{n_{\mathbb{H}}(\lambda, s)-m_{\lambda}}{s} d s
$$

Since $p(\lambda) \lesssim p(z)$, we will be done as soon as we prove that

$$
\int_{0}^{1} \frac{n_{\mathbb{H}}(\lambda, s)-m_{\lambda}}{s} d s \lesssim p(\lambda) .
$$

There exists $\delta>0$ (independent of $\lambda$ ) such that $D_{\mathbb{H}}(\lambda, \delta) \subset D(\lambda, p(\lambda))$. Then

$$
\begin{aligned}
\int_{0}^{\delta} \frac{n_{\mathbb{H}}(\lambda, s)-m_{\lambda}}{s} d s & =\sum_{0<\left|\frac{\lambda-\lambda^{\prime}}{\lambda-\lambda^{\prime}}\right|<\delta} m_{\lambda^{\prime}} \log \frac{\delta}{\left|\frac{\lambda-\lambda^{\prime}}{\lambda-\lambda^{\prime}}\right|} \leq \sum_{0<\left|\frac{\lambda-\lambda^{\prime}}{\lambda-\lambda^{\prime}}\right|<\delta} m_{\lambda^{\prime}} \log \frac{p(\lambda)}{\left|\lambda-\lambda^{\prime}\right|} \\
& \lesssim \sum_{0<\left|\lambda-\lambda^{\prime}\right|<p(\lambda)} m_{\lambda^{\prime}} \log \frac{p(\lambda)}{\left|\lambda-\lambda^{\prime}\right|} \leq N(\lambda, p(\lambda)) \lesssim p(\lambda) .
\end{aligned}
$$

For the remaining part we use condition (ii) in Theorem 1 and the estimate $\log t^{-1} \simeq 1-t$ for $\delta<t<1$. Taking $x=\operatorname{Re} \lambda$ we have

$$
\begin{aligned}
\int_{\delta}^{1} \frac{n_{\mathbb{H}}(\lambda, s)-m_{\lambda}}{s} d s & \lesssim \sum_{\lambda \neq \lambda^{\prime}} m_{\lambda^{\prime}} \frac{|\operatorname{Im} \lambda|\left|\operatorname{Im} \lambda^{\prime}\right|}{\left|\lambda-\bar{\lambda}^{\prime}\right|^{2}} \\
& \lesssim \sum_{\lambda \neq \lambda^{\prime}} m_{\lambda^{\prime}} \frac{|\operatorname{Im} \lambda|\left|\operatorname{Im} \lambda^{\prime}\right|}{\left|x-\lambda^{\prime}\right|^{2}} \lesssim|\operatorname{Im} \lambda| \simeq p(\lambda) .
\end{aligned}
$$

\section{REFERENCES}

[Bj66] G. Björck. Linear partial differential operators and generalized distributions, Ark. Mat. 6 (1966), 351-407. MR0203201 (34:3054)

[BrGa95] C.A. Berenstein and R. Gay. Complex Analysis and Special Topics in Harmonic Analysis, Springer-Verlag, 1995. MR 1344448 (96j:30001)

[BrLi95] C.A. Berenstein and B.Q. Li. Interpolating varieties for spaces of meromorphic functions, J. Geom. Anal. 5 (1995), 1-48. MR1315656 (96b:30064)

[EhMa74] L. Ehrenpreis and P. Malliavin. Invertible operators and interpolation in $\mathcal{A U}$ spaces, J. Math. pures appl. 53 (1974), 165-182. MR0402497 (53:6316)

[Ga81] J. Garnett. Bounded Analytic Functions, Academic Press, 1981. MR0628971 (83g:30037)

[Ho94] L. Hörmander. Notions of Convexity, Progr. Math., vol. 127, Birkhäuser, 1994. MR.1301332 (95k:00002)

[Le72] A.F. Leont'ev. On conditions of expandibility of analytic functions in Dirichlet series, Math. U.S.S.R. Izv. 6 (1972), 1265-1277. MR0335763 (49:543)

[OrSe99] J. Ortega-Cerdà and K. Seip. Multipliers for entire functions and an interpolation problem of Beurling, J. Funct. Anal. 162 (1999), 400-415. MR.1682065 (2000c:30071)

[Ou03] M. Ounaïes. On interpolating discrete varieties for weighted spaces of entire functions, Anal. Math. 29 (2003), 59-74. MR:1965313(2003m:32005) 
[Sq81] W.A. Squires. Necessary conditions for universal interpolation in $\hat{\mathcal{E}}^{\prime}$, Canad. J. Math. 33 (1981), 1356-1364. MR0645231 (83g:30040)

[Sq83] W.A. Squires. Geometric condition for universal interpolation in $\hat{\mathcal{E}}^{\prime}$, Trans. Amer. Math. Soc. 280 (1983), 401-413. MR0712268 (84h:30058)

Departament de Matemàtica Aplicada i Anàlisi, Universitat de Barcelona, Gran Via 585, 08071-BARCElona, SPAin

E-mail address: xavier.massaneda@ub.edu

Departament de Matemàtica Aplicada i Anàlisi, Universitat de Barcelona, Gran Via 585, 08071-Barcelona, Spain

E-mail address: jortega@ub.edu

Institut de Recherche Mathématique Avancée, Université Louis Pasteur, 7 Rue René Descartes, 67084 Strasbourg Cedex, France

E-mail address: ounaies@math.u-strasbg.fr 